\title{
Unordnung und Unsicherheit in großstädtischen Wohngebieten - Die überschätzte Rolle von „Broken Windows" und die Herausforderungen ethnischer Diversität
}

\author{
Dietrich Oberwittler • Heleen Janssen • Dominik Gerstner
}

Online publiziert: 6. Oktober 2017

(C) Der/die Autor(en) 2017. Dieser Artikel ist eine Open-Access-Publikation.

Zusammenfassung Zeichen physischer und sozialer Unordnung im öffentlichen Raum gelten im „Broken Windows“-Ansatz als eine maßgebliche Ursache für Unsicherheitsgefühle und den dadurch verstärkten Niedergang großstädtischer Wohngebiete. Die empirische Bestätigung für diese These ist jedoch schwach, da viele Studien auf subjektiven Wahrnehmungen basieren, welche mit dem Unsicherheitsempfinden eng korreliert sind. Wir untersuchen anhand unabhängiger und systematischer Beobachtungen, welche individuellen und sozialräumlichen Eigenschaften den subjektiven „Wahrnehmungsfilter“ der Bewohner*innen prägen und wie stark die Wirkung von Unordnung auf das Unsicherheitsgefühl tatsächlich ist. Die Auswertungen basieren auf der Bewohner*innenbefragung des Projekts SENSIKO in Köln und Essen mit ca. 6500 Befragten in 140 Wohngebieten sowie auf systematischen sozialen Beobachtungen und Strukturdaten. Es zeigt sich in Übereinstimmung mit früheren Studien, dass die Wahrnehmung sowohl von Unordnung als auch von Unsicherheit maßgeblich vom Anteil der sichtbaren Minderheiten angetrieben wird. Erstmals wird gezeigt, dass dieser Effekt sehr stark von den individuellen Einstellungen der Bewohner*innen zu Migration abhängig ist. Das Ausmaß systematisch beobachteter Unordnung hat über den Effekt der ethnischen Diversität hinaus nur in Essen, nicht aber in Köln einen furchtsteigernden Effekt. Die Ergebnisse unterstreichen die Herausforderung, die dem Zusammenleben in Großstädten durch eine wachsende Migration erwächst, und weisen zugleich auf die starke Kontextabhängigkeit der Wirkungen sozialräumlicher Problemlagen auf das lokale Unsicherheitsempfinden hin.

D. Oberwittler $(\bowtie) \cdot$ D. Gerstner

Max-Planck-Institut für ausländisches und internationales Strafrecht,

Günterstalstraße 73, 79100 Freiburg i. Br., Deutschland

E-Mail: d.oberwittler@mpicc.de

H. Janssen

Delft University of Technology, Delft, Niederlande 


\title{
Disorder and Perceptions of Insecurity in Urban Neighbourhoods - The Overrated Role of "Broken Windows" and the Challenge of Ethnic Diversity
}

\begin{abstract}
According to the "broken windows"-approach, signs of physical and social disorder in public spaces are a major cause of insecurity perceptions and the subsequent decline of urban neighbourhoods. Yet, the empirical support is weak as many studies have relied on subjective perceptions of disorder which are closely correlated with fear of crime. Using independent and systematic social observations we investigate the individual and ecological factors influencing the 'perception bias' of disorder, and ask how strong the impact of disorder on fear actually is. Our analyses are based on a survey of ca 6500 respondents and systematic social observations in 140 neighbourhoods in Cologne and Essen. We show that both the perception of disorder and fear of crime are mainly driven by the percentage of visible minorities. For the first time, we show that this effect is strongly dependent on individual attitudes towards migration. Only in one city, Essen, does the amount of systematically observed disorder have a fear-inducing effect over and above the effect of ethnic diversity. These results highlight the challenges of increased migration for social cohesion, and at the same time pinpoint at the importance of social contexts for the impact of social problems on feelings of insecurity.
\end{abstract}

\section{Einleitung}

Warum und wovor haben Menschen Angst, wenn es um das schwer fassbare Phänomen Kriminalitätsfurcht geht? Eine lange Forschungsgeschichte hat viele Dimensionen und Zusammenhänge beleuchtet, die von Persönlichkeitsmerkmalen bis zu Wohlfahrtsregimen reichen (Hale 1996; Hirtenlehner und Hummelsheim 2015; Hummelsheim et al. 2011). Dass Bewohner*innen in Großstädten und dort besonders in einigen Wohngebieten mehr Kriminalitätsfurcht äußern als anderswo, hat die Bedeutung urbaner Lebensumwelten als einen wichtigen Erklärungsansatz in den Vordergrund gerückt (Brunton-Smith und Jackson 2012; Lewis und Maxfield 1980). Unsicherheitswahrnehmungen speisen sich nicht vorrangig aus ,tatsächlichen“ Kriminalitätsrisiken, sondern aus einem schwer zu entwirrenden Komplex aus verschiedenen Ängsten, persönlichen Verletzlichkeiten und Merkmalen des städtischen Raumes und insbesondere des engeren Wohnumfelds. Besonders einflussreich, aber nicht unumstritten ist die „Broken Windows“-Theorie mit ihrer Behauptung, dass physische und soziale Unordnung im öffentlichen Raum mehr als tatsächliche Straftaten Furcht erzeugt und Bewohner*innen verunsichert - und damit den Boden für mehr Kriminalität bereitet (Kelling und Wilson 1982). Den gleichen Fokus setzte Wesley Skogan (1990) in seinem einflussreichen Buch „Disorder and Decline“. Auch wenn diese These umstritten bleibt, haben fast alle größeren Studien zu urbaner Kriminalität seit den 1980er-Jahren die Rolle von Unordnung untersucht (Skogan 2015).

Angesichts ihrer Popularität ist es jedoch erstaunlich, wie schwach die empirische Bestätigung der „Broken Windows“-Theorie ist (Harcourt 2005; Link et al. 
2014). Als ein zentrales Problem hat sich dabei die Messung der Unordnung erwiesen, die in den meisten Studien durch Bewohner*innenbefragungen erfolgte. Schon früh kam der Verdacht auf, dass ihre Bedeutung überschätzt wird, wenn man sowohl Unordnung als auch diejenigen sozialen Prozesse, die nach der Theorie durch sie ausgelöst werden - insbesondere das Unsicherheitsempfinden - in der gleichen Befragung misst (Covington und Taylor 1991). Daher führten einige Studien systematische soziale Beobachtungen (systematic social observations, SSO) als eine unabhängige und objektivere Messung von Unordnung durch und stellten damit in der Regel schwächere Beziehungen zwischen Unordnung und sozialen Wahrnehmungen und Prozessen fest (Häfele 2013; Perkins und Taylor 1996; Skogan 2015). Während durch SSO gemessene Unordnung näher am ,objektiven“ Zustand städtischer Räume ist, fehlt ihr jedoch die soziale Bedeutung in der subjektiven Wahrnehmung der Bewohner*innen, ohne die Unordnung im Sinne des Thomas-Theorems letztlich keine sozialen Auswirkungen haben kann (Kubrin 2008; Merton 1948). Daher sind die subjektiv gefärbten Wahrnehmungen der Bewohner*innen selbst ein ,bedeutsamer Aspekt des sozialen Lebens in Wohngebieten" (Sampson 2009, S. 24), und ihre Diskrepanz zur objektiv gemessenen Unordnung, die als „Wahrnehmungsverzerrung“ (perception bias) bezeichnet wird, wurde zu einem eigenen Forschungsthema.

In ihrer grundlegenden Studie in Chicago deckten Sampson und Raudenbush (2004) auf, dass unabhängig vom objektiv gemessenen Ausmaß der Unordnung die soziodemographische Struktur der Stadtviertel und vor allem die Armutskonzentration und der Anteil der Afro-Amerikaner*innen die Wahrnehmungen entscheidend beeinflussten, was sie auf implizite ethnische Stereotype zurückführten und als Ausdruck einer kollektiven Stigmatisierung von Stadtvierteln werteten. Eine isolierte Betrachtung von Unordnung ignoriert demnach, dass soziale Wahrnehmungen eingebettet sind in komplexere sozialräumliche Kontexte und dass subjektive Wahrnehmungsfilter bei der Bewertung scheinbar ,objektiver“ Raummerkmale eine wichtige Rolle spielen.

Wir benutzen Daten einer systematischen sozialen Beobachtung von Unordnung gemeinsam mit Befragungsdaten und amtlichen Strukturdaten aus 140 großstädtischen Wohngebieten, um (1) nach dem Vorbild von Sampson und Raudenbush (2004) sozialräumliche und individuelle Einflussfaktoren auf die Wahrnehmungsverzerrung von Unordnung zu untersuchen und (2) die Bedeutung von Unordnung für das Unsicherheitsgefühl in Wohngebieten im Kontext anderer sozialräumlicher Merkmale und individueller Einstellungen zu hinterfragen. Für beide Aspekte spielen ethnische Diversität und Einstellungen zu Migration eine entscheidende, bislang wenig berücksichtigte Rolle.

\footnotetext{
1 Wörtliche Zitate in diesem Artikel wurden von den Autor*innen ins Deutsche übersetzt.
} 


\section{Theorie und Forschung}

\subsection{Städtische Unordnung im Modell der sozialen Desorganisation: Ein zentraler Faktor für Unsicherheit?}

Nach Wesley Skogan (2015, S. 465) ist Unordnung im öffentlichen Raum ein wichtiger Mechanismus in der Entwicklung von sozialräumlichen Problemen. Im so genannten „systemischen Modell“ der sozialen Desorganisationstheorie entspricht dies einem negativen Feedback-Effekt von abweichendem Verhalten auf den sozialen Zusammenhalt und die soziale Organisation von Nachbarschaften (die wiederum zur Kontrolle abweichenden Verhaltens wichtig ist) (Brunton-Smith et al. 2014; Oberwittler 2013; Taylor 2001). Der Ausdruck „Unordnung“ (disorder) ebenso wie der schwer ins Deutsche übersetzbare Begriff Incivilities bezeichnen verschiedene Verhaltensweisen und deren sichtbare physische Spuren, die die Regeln ,zivilisierten" Verhaltens in der Nachbarschaft verletzen. Der Soziologe Albert Hunter (1978) prägte den Begriff Incivilities in Abgrenzung zu Kriminalität und behauptete, dass Incivilities, da sie dauerhafte Spuren hinterlassen, letztlich stärkere negative Wirkungen hätten als kriminelle Handlungen. ,[Incivilities] vermitteln [...] den Verlust ziviler Gesellschaft [und] erzeugen Angst und Unsicherheit unter den Stadtbewohnern" (Hunter 1978, S. 7). Daran anknüpfend behaupteten Kelling und Wilson (1982) und Skogan (1990) eine Kausalkette, nach der Unordnung Unsicherheitsgefühle auslöst, die zur Schwächung des Zusammenhalts und der informellen Sozialkontrolle beitragen, was wiederum Kriminalität erleichtert. ${ }^{2}$ Empirische Hinweise auf diese Kausalkette ergaben sich beispielsweise in einer Analyse des British Crime Survey durch Markowitz et al. (2001, S. 310): „Kohäsion verringert Unordnung, was Furcht steigert, was Kohäsion vermindert. Der dominante Effekt in diesem Kreis ist der Effekt von Unordnung auf Furcht." Schematisch ist diese Kausalkette in Abb. 1 dargestellt. Die schwarz dargestellten Elemente dieses Modells sollen in diesem Aufsatz empirisch getestet werden (soweit dies mit Querschnittsdaten möglich ist), während die grau gezeichneten Elemente jenseits unserer Analysen liegen. Der „Wahrnehmungsfilter“ in Abb. 1 reflektiert die im ursprünglichen „Broken Windows“-Ansatz fehlende und durch viele empirischen Studien gereifte Vorstellung, dass sich objektiv messbare strukturelle Problemlagen und sichtbare Unordnung

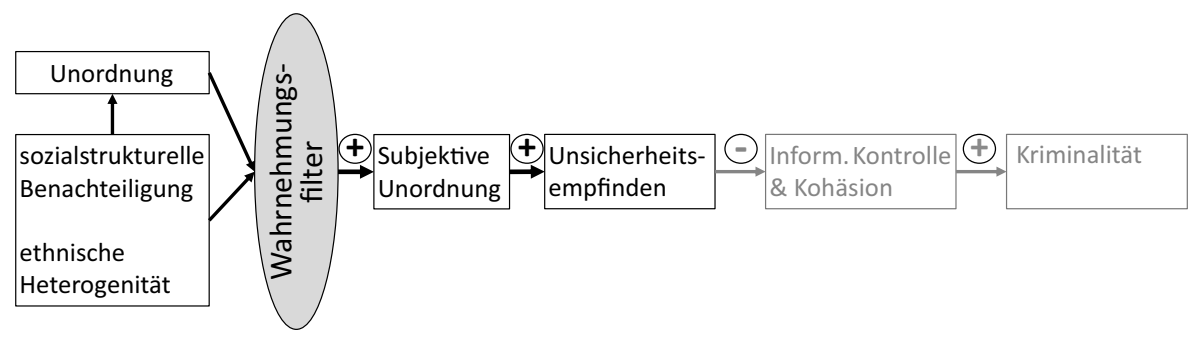

Abb. 1 Schema des „Broken Windows“-Ansatzes (erweitert)

\footnotetext{
2 An zweiter Stelle wird auch ein direkter Effekt auf potenzielle Täter*innen angenommen, denen signalisiert werde, dass Regelverletzungen toleriert würden.
} 
nicht unmittelbar in Unsicherheit umwandeln, sondern nur vermittelt durch subjektive und damit auch individuell unterschiedliche Wahrnehmungsprozesse Wirkungen entfalten, die in Analysen berücksichtigt werden sollten (Kubrin 2008).

Die Verbindung von Unordnung und Unsicherheitswahrnehmungen ist also ein Schlüsselelement der „Broken-Windows“-Theorie. In vielen Studien fanden sich starke Zusammenhänge zwischen beiden Konzepten (Brunton-Smith et al. 2014; Drakulich 2013; Häfele 2013; LaGrange et al. 1992; Mellgren et al. 2011; Oberwittler 2008; Ross and Jang 1999; Wyant 2008). Aber schon sehr früh wiesen Ralph Taylor und seine Kolleg*innen auf die subjektiven Verzerrungen der Wahrnehmungen hin. Innerhalb desselben Wohngebiets sahen Befragte, die sich unsicherer fühlten, auch mehr Unordnung (Perkins et al. 1992; Perkins und Taylor 1996; Robinson et al. 2003; Taylor et al. 1985; Lewis und Maxfield 1980; Link et al. 2014). Das ist ein Hinweis auf eine mögliche Messfehlerkorrelation und stellt die kausalen $\mathrm{Zu}$ sammenhänge zwischen Unordnung und Unsicherheit in Frage (Brunton-Smith und Sturgis 2011; Skogan 2015).

Angesichts dieser methodischen Zweifel war es sehr wichtig, das Ausmaß der Unordnung im öffentlichen Raum unabhängig von Bewohner*innenbefragungen durch eigene systematische Beobachtungen zu erheben. Hier sind das „Project on Human Development in Chicago Neighborhoods“ (Sampson und Raudenbush 1999), die von Ralph Taylor geleitete Längsschnittstudie in Baltimore (Robinson et al. 2003; Taylor 2001) sowie in Deutschland die von Christian Lüdemann geleitete Hamburger Studie „Incivilities, Sozialkapital und Kriminalität“ (Häfele 2013; Lüdemann 2006) zu nennen. Diese Studien korrigierten nicht nur übertriebene Annahmen zu „Broken Windows“, sondern öffneten auch die Tür zum Verständnis der Faktoren, die die subjektiven Raumwahrnehmungen der Bewohner*innen beeinflussen und machten damit eine weitere wichtige Dimension sozialer Prozesse in Wohngebieten zugänglich (Brunton-Smith und Sturgis 2011).

\subsection{Die Diskrepanz zwischen ,objektiver" und ,subjektiver" Unordnung und ihre Hintergründe}

Die Frage, wie ähnlich oder verschieden objektive Beobachtungen und subjektive Wahrnehmungen von Unordnung sind, gleicht der Frage, ob ein Glas halbvoll oder halbleer ist. Skogan (2015, S. 478) spricht von einem ,,vernünftigen Maß an Übereinstimmung“ zwischen den beiden Methoden, aber in keiner Studie lag der Anteil der geteilten Varianz bei mehr als $50 \%$. Es bleibt ein großer Raum für andere Einflüsse. Hierfür ist die Annahme zentral, dass bei Wahrnehmungen von Unordnung eine allgemeine und eher diffuse Empfindung urbaner Gefahren mitschwingt. Die enge Verbindung von wahrgenommener Unordnung und Kriminalitätsfurcht auf individueller Ebene zeigt, dass diejenigen, die aufgrund sozialer und psychologischer Verletzlichkeiten furchtsamer sind, auch Zeichen der Unordnung intensiver und bedrohlicher wahrnehmen und daher mehr Unordnung ,sehen“. Die Forschung zu den ,expressiven“ Funktionen von Kriminalitätsfurcht hat gezeigt, dass besonders bei Menschen, die sich eher macht- und schutzlos fühlen, Kriminalität eine starke symbolische Funktion für grundlegende soziale Sorgen hat (Farrall et al. 2009; Hirtenlehner 2006; Hirtenlehner et al. 2016; Jackson 2004, 2009). Hirtenlehner und 
Farrall (2013, S. 17) untersuchten die wahrgenommene Unordnung zusammen mit Kriminalitätsfurcht und verschiedenen anderen Sorgen in einem Strukturgleichungsmodell und kamen zu dem Ergebnis, ,dass die bekannte Beziehung von Sorgen über Unordnung und Kriminalität größtenteils auf ihre gemeinsame Färbung durch ein allgemeines und amorphes Gefühl der Unsicherheit“" zurückgeht. Auch Eifler et al. (2009) stellten eine Verbindung zwischen dem wahrgenommenen Opferrisiko und höherer Sensibilität gegenüber urbaner Unordnung fest.

In diesem Zusammenhang spielt ethnische Differenz eine besondere Rolle. Auf der individuellen Ebene berichteten Befragte in US-amerikanischen Studien, die einer ethnischen Minderheit angehören, weniger Unordnung als ihre Nachbar*innen (Drakulich 2013; Franzini et al. 2008; Hipp 2010; Sampson und Raudenbush 2004). Als ein kollektives Merkmal der Wohngebiete jedoch war die Konzentration ethnischer Minderheiten ebenso wie die Armutskonzentration ein starker Prädiktor für die Wahrnehmungsverzerrung. In Chicagoer Wohngebieten mit höherem Anteil Schwarzer und Latinos berichteten Bewohner*innen mehr Unordnung relativ zur objektiv gemessenen Unordnung (Sampson und Raudenbush 2004; vgl. Drakulich 2013). In einem Laborexperiment in Taiwan wurden Fotos von Straßenszenen mit Migrant*innen als unordentlicher bewertet als die gleichen Straßenszenen mit einheimischen Personen (wobei die Kleidung jedes Mal auf einen niedrigen Sozialstatus hindeutete) (Yang und Pao 2015).

Gleichgerichtete Effekte fanden sich auch in vielen Studien zu den sozialräumlichen Bedingungen von Kriminalitätsfurcht ebenso wie von Sozialkapital: Auch unter Kontrolle weiterer Faktoren ist Kriminalitätsfurcht in Wohngebieten mit einem größeren Bevölkerungsanteil ethnischer Minderheiten höher und das Vertrauen und die soziale Kohäsion niedriger als anderswo (Chiricos et al. 2001; Fitzgerald et al. 2012; Hooghe et al. 2016; Oberwittler und Gerstner 2016; Pickett et al. 2012; Pritsch und Oberwittler 2015; Quillian und Pager 2001; Semyonov et al. 2012; van der Meer und Tolsma 2014; Visser et al. 2013). Bislang bildeten lediglich Studien aus dem ,superdiversen“ London eine Ausnahme (Sturgis et al. 2013). Die Fülle dieser empirischen Belege lässt nur den Schluss zu, dass Wahrnehmungen von Kriminalitätsgefahren, Unordnung und sozialem Zusammenhalt gemeinsam und grundlegend durch ethnische Diversität im Wohngebiet beeinflusst werden, die wiederum in den meisten Ländern untrennbar mit sozialer Benachteiligung und Segregation verbunden sind.

Vermutlich bilden tief verwurzelte ethnozentrische Einstellungsmuster die kognitive Basis dieser Zusammenhänge (Chiricos et al. 2001; Quillian und Pager 2001; Sampson und Raudenbush 2004; Sampson 2009; Skogan 1995; Wickes et al. 2013). Der enge Zusammenhang von (offener) Fremdenfeindlichkeit und Kriminalitätsfurcht wurde wiederholt konstatiert (Fitzgerald et al. 2012; Golden 2012; Hirtenlehner et al. 2016; Oberwittler und Gerstner 2016; Sessar 2010; Sessar et al. 2007). Auf die gemeinschaftsstiftenden Funktionen der Ausgrenzung von Abweichler*innen und Außenseiter*innen haben in Anlehnung an Emile Durkheim bereits George H. Mead (1918) und Kai Erikson (1966) hingewiesen. Der Prototyp des ,kriminellen Ausländers" bedient diese symbolische Funktion besonders gut und ist daher auch ein „Klassiker“ in rechtspopulistischen Kampagnen (Albrecht 2002; Smith 2010). 
Unsicherheitsgefühle angesichts ethnischer Diversität sind jedoch nicht auf Menschen mit tendenziell fremdenfeindlichen Einstellungen beschränkt. In der Sozialpsychologie wird zwischen expliziten und impliziten Einstellungen unterschieden; letztere wirken unbewusst, sind schwer zu messen und trotzdem sehr wirkungsmächtig (Dovidio et al. 2016; Kawakami et al. 2017). Mithilfe eines „Implicit Association Test“" konnte Drakulich (2015) zeigen, dass implizite ethnische Stereotype einen stärkeren Effekt auf Unsicherheitswahrnehmungen hatten als explizite, da letztere durch den Effekt der sozialen Erwünschtheit unterdrückt werden können.

Eine einfach zu klärende Frage ist, ob Befragte aus Mehrheits- und Minderheitsgruppen unterschiedlich auf ethnische Diversität im Wohngebiet reagieren. Sampson und Raudenbush (2004) zeigten in Chicago, dass die subjektive Wahrnehmung von Unordnung von weißen und schwarzen Befragten gleichermaßen durch den schwarzen Bevölkerungsanteil beeinflusst wurde und die der Latinos sogar noch mehr. Diese Gleichförmigkeit der Wahrnehmungsverzerrung spiegelt die Ergebnisse eines Videoexperiments wieder, in dem weiße und schwarze Betrachter*innen gleichermaßen schwarze Personen im Video als gefährlicher einstuften als weiße (Correll et al. 2002; vgl. Kawakami et al. 2017). Im Gegensatz dazu berichteten Schwarze in Londoner Stadtvierteln mit höherer ethnischer Diversität weniger Kriminalitätsfurcht, entgegen dem Trend aller übrigen ethnischen Gruppen (Brunton-Smith and Sturgis 2011).

Insgesamt unterstreichen diese Forschungsergebnisse die Bedeutung der ethnischen Diversität in urbanen Wohngebieten sowohl für die Wahrnehmungen von Unordnung als auch von Unsicherheit; beide Wahrnehmungen sind scheinbar eng miteinander verwoben und lassen sich nicht einfach auf „objektive“ Risiken zurückführen. Robert Sampson (2009) hat jedoch auf die „realen“ Konsequenzen der subjektiv gefärbten Wahrnehmungen von sozialräumlichen Problemen hingewiesen. Über die soziale Reputation - und damit auch über die zukünftige Entwicklung - von Stadtvierteln entscheidet nicht das objektive Ausmaß von Unordnung und Kriminalität, sondern kollektive, subjektiv gefärbte Wahrnehmungen der Menschen gemäß dem so genannten „Thomas-Theorem“, nachdem als real wahrgenommene Situationen real in ihren Konsequenzen sind (Merton 1948). Eine Vernachlässigung des subjektiven Charakters sozialräumlicher Wahrnehmungen würde die sozialen Prozesse in urbanen Wohngebieten grob vereinfachen.

\section{Fragestellungen}

Vor diesem theoretischen Hintergrund ist es das erste Ziel dieses Aufsatzes, maßgebliche Einflussfaktoren der Wahrnehmungsverzerrung städtischer Unordnung herauszuarbeiten. Wir konzentrieren uns dabei zum einen auf die Einflüsse der soziodemographischen Struktur und Kriminalitätsbelastung von Stadtvierteln. Übereinstimmend mit den Ergebnissen bisheriger Studien rechnen wir besonders mit einem Effekt der ethnischen Diversität auf die Wahrnehmungsverzerrung. Diese Studien stammen jedoch ausschließlich aus den USA mit ihren besonderen „race relations“ (Richeson und Sommers 2016), was die Übertragbarkeit auf europäische Länder als nicht selbstverständlich erscheinen lässt. In der Hamburger Studie wurden einzelne 
sozialräumliche Merkmale nicht getrennt untersucht, sondern soziale und ethnische Segregation in einem Faktor zusammengefasst (Häfele 2013). Die Wirkung ethnischer Diversität wurde bislang zudem noch nie im Kontext der Frage untersucht, ob und wie stark sie von den expliziten ethnischen Vorurteilen der Bewohner*innen abhängig ist, wie wir es hier mit Hilfe einer Skala zu xenophoben Einstellungen machen. Wir nehmen an, dass Befragte mit tendenziell xenophoben Einstellungen eine stärkere Wahrnehmungsverzerrung zeigen als andere Befragte.

Das zweite Ziel des Aufsatzes ist die Klärung des Einflusses von Unordnung auf die Unsicherheitswahrnehmungen im Wohngebiet, also einer zentralen theoretischen Annahme der „Broken Windows“-Theorie. Da bereits bekannt ist, dass subjektiv wahrgenommene Unordnung und Unsicherheitsgefühle gemeinsam durch persönliche Einstellungsmuster und Verletzlichkeiten geprägt werden und daher eng verwoben sind, analysieren wir hier vorrangig die Effekte der durch SSO gemessenen Unordnung und erwarten keine oder geringe Effekte, also eine Zurückweisung der „Broken Windows“-Theorie.

\section{Daten und Methoden}

Wir verwenden die Daten der postalischen Bewohner*innenbefragung aus dem Projekt „Sicherheit älterer Menschen im Wohnquartier“ (SENSIKO), die 2014 und 2015 in 140 zufällig ausgewählten Wohngebieten in Köln und Essen durchgeführt wurde (Gerstner und Oberwittler 2016). ${ }^{3}$ An der ersten Welle nahmen 6565 zufällig aus dem Einwohnermelderegister ausgewählte Bewohnerinnen und Bewohner in Privathaushalten im Alter von 25 bis 89 Jahren teil, wobei die Altersgruppe 60 bis 89 Jahre wegen eines besonderen Fokus des Projekts auf Ältere etwa zweifach übergewichtet wurde. Die Rücklaufquote betrug $41 \%$ und war bei Älteren und deutschen Staatsangehörigen höher als bei Jüngeren und Nichtdeutschen. Die durchschnittliche Anzahl von Befragten pro Wohngebietwar 44, nur in vier Wohngebieten wurden weniger als 30 Bewohner*innen befragt (Minimum 22). Der Zusammenhang von $r=0,87$ auf der Wohngebietsebene zwischen amtlicher Quote der Empfänger*innen staatlicher Unterstützungsleistungen und dem Anteil der Befragten, die solche Unterstützungen berichten, sowie der Zusammenhang von $r=0,86$ zwischen dem amtlichen Anteil der Bewohner*innen mit Migrationshintergrund (nur in Köln verfügbar) und dem Anteil der Befragten mit Migrationshintergrund deuten auf eine gute soziodemographische Abbildqualität der Stichprobe hin. Im Herbst 2015 erfolgte eine Wiederholungsbefragung derselben Befragten, an der sich noch 3745 Personen beteiligten (57\% der Befragten der ersten Welle). Die Analysen in diesem Aufsatz basieren mit Ausnahme der zusätzlichen Items zur wahrgenommenen Unordnung ausschließlich auf der ersten Befragungswelle im Frühjahr 2014. Querschnittsanalysen unterliegen Einschränkungen hinsichtlich der kausalen Interpretation von Zusammenhängen (Taylor 2015). Häufiger werden Effekte aufgrund von wechselseitiger Kausalität (Endogenität) überschätzt; findet man jedoch keine

\footnotetext{
${ }^{3}$ Dieser Methodenbericht zur Befragung mit weitergehenden Informationen ist abrufbar unter https:// www.mpicc.de/de/forschung/forschungsarbeit/kriminologie/sensiko.html.
} 
oder sehr schwache Zusammenhänge, ist es sehr unwahrscheinlich, dass diese in längsschnittlicher Analyse stärker werden. Die Befragungsdaten werden mit amtlichen Daten zur soziodemographischen Struktur der Wohngebiete, mit polizeilichen Daten zur Häufigkeit verschiedener Kriminalitätsformen und mit den systematisch erhobenen Beobachtungen von Unordnung verknüpft.

Die subjektiv wahrgenommene Unordnung wurde durch 12 Fragen nach der Häufigkeit der Beobachtung physischer und sozialer Incivilities gemessen, die auf die beiden Befragungswellen verteilt waren. Aufgrund der Ergebnisse einer konfirmatorischen Faktorenanalyse wurde eine übergreifende, eindimensionale Skala für alle Teilnehmenden der ersten Befragungswelle gebildet; das heißt, dass physische und soziale Incivilities in der Wahrnehmung der Bewohner*innen eine gemeinsame Dimension von Unordnung bilden. ${ }^{4}$ Der durch die Intraklassenkorrelation (ICC) gemessene Anteil der geteilten Varianz zwischen Befragten derselben Wohngebiete liegt mit $32 \%$ sehr hoch. Die Bewohner*innen stimmen in ihren Wahrnehmungen also deutlich überein. Das wohngebietsbezogene Unsicherheitsgefühl wurde durch zwei Fragen zum Sicherheitsgefühl gemessen, wenn man ,nach Einbruch der Dunkelheit“ bzw. „tagsüber alleine zu Fuß im eigenen Wohngebiet unterwegs“ ist, die mit $r=0,61$ miteinander korrelieren. Auch beim Unsicherheitsgefühl liegt der ICC mit $16 \%$ noch recht hoch.

Die materielle Lage wurde durch zwei Fragen zur subjektiven Einkommenslage sowie einer Frage zum Empfang staatlicher Unterstützungsleistungen gemessen; höhere Werte signalisieren eine prekäre finanzielle Lage. Die Skala xenophobe Einstellungen basiert auf vier Items wie zum Beispiel „Die Zuwanderer aus ärmeren Ländern sind eine Belastung für das soziale Netz in Deutschland" (Cronbach's Alpha 0,72). Viktimisierung basiert auf einer Fragebatterie zu persönlichen Opfererfahrungen während der letzten zwei Jahre; sechs Items betreffen Eigentumsdelikte (Wohnungseinbruch, Sachbeschädigung, Diebstahl, Betrug), drei Items betreffen Gewalterfahrungen (Körperverletzung, Bedrohung, sexuelle Belästigung).

Die systematischen sozialen Beobachtungen der physischen und sozialen Unordnung wurden 2015 durch geschulte Studierende in allen 140 Wohngebieten durchgeführt. Dazu wurden in jedem Gebiet zufällig durchschnittlich drei Straßenkreuzungen als „Knotenpunkte“ und alle Straßenabschnitte, die einen Radius von $200 \mathrm{~m}$ um die Kreuzungen schneiden, ausgewählt. Diese Stichprobe umfasste etwa $40 \%$ aller Straßenabschnitte in den Stadtvierteln der Bewohnerbefragung. In jedes Wohngebiet wurden zwei verschiedene Beobachter*innen geschickt, die sich das Gebiet aufteilten. Sie notierten die Häufigkeit von je 17 physischen und sozialen Incivilities sowie Merkmale der Baustruktur, Infrastruktur und des Verkehrs. Zur Orientierung und Datenerhebung hatten die Studierenden einen Tablet-PC mit digitalen Stadtplänen und GPS-Ortung dabei. Die Einzelitems wurden zu Indizes aufsummiert, anhand der Länge der Straßenabschnitte standardisiert und wegen der sehr schiefen Verteilung log-transformiert. Ein Test auf Interrater-Reliabilität durch Doppelbeobachtungen

\footnotetext{
${ }^{4}$ Mplus schätzt mit dem Verfahren der „full information maximum likelihood estimation“ gültige Werte auch für diejenigen Befragten, die nicht mehr an der zweiten Welle teilgenommen haben. Der Wortlaut dieser und aller folgenden Surveyfragen und die Eigenschaften der gebildeten Skalen sind abrufbar unter https://www.mpicc.de/de/forschung/forschungsarbeit/kriminologie/sensiko.html.
} 
ergab eher niedrige Werte von Krippendorff's Alpha zwischen 0,50 und 0,40 für leerstehende Gebäude, Graffiti und Aufkleber und noch niedrigere Werte für andere Items. Wie erwartet war die Reliabilität für soziale Incivilities besonders schlecht, da diese Phänomene selten auftreten und ihre Beobachtung zeitabhängig ist. Bei dieser Reliabilitätsanalyse auf der kleinsten räumlichen Ebene der Straßenabschnitte ist mit einer hohen Zufallsstreuung zu rechnen. Auf der nächsthöheren Ebene der Straßennetzwerke betrug die Korrelation des Summenindex der physischen Unordnung zwischen zwei Doppelbeobachtungen $r=0,71$ und die entsprechende Korrelation der kommerziellen Infrastruktur $r=0$,96. Auch die im Vergleich zu bisherigen Studien hohe Korrelation von $r=0,72$ zwischen der durch SSO gemessenen und der durch die Befragten subjektiv wahrgenommenen physischen Unordnung auf der Wohngebietsebene sprechen für die Datenqualität. Die entsprechenden Werte lagen in Chicago bei $r=0,55$ (Raudenbush and Sampson 1999, S. 623) und in Hamburg bei $r=0,61$ (Häfele 2013, S. 165).

Als Strukturmerkmale der Wohngebiete werden offizielle Daten der statistischen Ämter von Köln und Essen verwendet. Der Anteil ethnischer Minderheiten wird anhand der nicht-deutschen Staatsangehörigkeit gemessen, da das Merkmal Migrationshintergrund nicht in Essen verfügbar war (die Korrelation zwischen beiden in Köln beträgt $r=0,92$ ). Wir unterscheiden zwischen EU- und Nicht-EU-Herkunftsländern; letztere stellen eine Proxy-Variable für sichtbare ethnische Minderheiten dar (Richeson und Sommers 2016). Konzentrierte Armut wird durch den Anteil der Unterstützungsempfänger*innen nach SGB II (hauptsächlich ALG II) und XII (Sozialhilfe) gemessen. Polizeiliche Einzelfalldaten verschiedener Delikte wurden geocodiert, räumlich aggregiert und es wurden bevölkerungsbezogene Raten gebildet. Da in den folgenden Mehrebenenmodellen die Prädiktoren auf der Ebene der Wohngebiete standardisiert wurden, lassen sich ihre Einflussstärken direkt miteinander vergleichen. Die deskriptive Statistik der in den folgenden Regressionsmodellen verwendeten Variablen ist im Anhang dokumentiert.

\section{Ergebnisse I: Einflussfaktoren der Wahrnehmungsverzerrung}

Im ersten Schritt analysieren wir mithilfe linearer Mehrebenenmodelle die Abweichungen der subjektiven Wahrnehmungen von Unordnung durch die Bewohner*innen von den systematischen Beobachtungen der geschulten Beobachter*innen in Abhängigkeit von strukturellen Merkmalen der Wohngebiete, unter anderem der ethnischen Diversität sowie der xenophoben Einstellungen der individuellen Befragten (Janssen et al. in Vorbereitung). Die subjektive Wahrnehmung von Unordnung als abhängige Variable wird in Tab. 1 (Modell 1) auf der individuellen Ebene (L1) durch soziodemographische Merkmale und Opfererfahrungen erklärt. Da auf der Wohngebietsebene für die durch SSO gemessene physische und soziale Unordnung kontrolliert wird (wodurch sich die Varianz zwischen den Wohngebieten bereits um $55 \%$ reduziert), erklären die übrigen Prädiktoren die Differenz zwischen ,objektiver“ und „subjektiver“ Unordnung, die wir als Wahrnehmungsverzerrung bezeichnen.

Innerhalb der Wohngebiete nehmen Befragte mit niedrigerem Bildungsstatus, schlechterer materieller Lage, ohne volle Erwerbstätigkeit und solche mit mehr als 
Tab. 1 Lineare Mehrebenen-Regression der wahrgenommenen Unordnung im Wohngebiet (Köln und Essen)

\begin{tabular}{|c|c|c|c|c|}
\hline & \multicolumn{2}{|l|}{ Modell 1} & \multicolumn{2}{|l|}{ Modell 2} \\
\hline & $\mathrm{B}$ & SE & $\mathrm{B}$ & SE \\
\hline \multicolumn{5}{|l|}{ L1 $(N=6136$ Befragte $)$} \\
\hline Alter (in Dekaden) & $0,124 * *$ & 0,043 & $0,119 * *$ & 0,043 \\
\hline Alter quadriert & $-0,013 * * *$ & 0,004 & $-0,012 * *$ & 0,004 \\
\hline \multicolumn{5}{|l|}{ Migrationshintergrund (Ref. nein) } \\
\hline Europäische Herkunft & $-0,048$ & 0,037 & $-0,054$ & 0,037 \\
\hline Nicht-europäische Herkunft & $-0,067 *$ & 0,034 & $-0,087 *$ & 0,034 \\
\hline \multicolumn{5}{|l|}{ Schulbildung (Ref.: Mittlere Reife) } \\
\hline Kein Abschluss & $0,132 *$ & 0,060 & $0,132 *$ & 0,060 \\
\hline Volks-/Hauptschule & $0,083 * *$ & 0,027 & $0,081 * *$ & 0,027 \\
\hline (Fach-)Abitur & $-0,022$ & 0,033 & $-0,018$ & 0,033 \\
\hline (Fach-)Hochschule & $-0,064 *$ & 0,029 & $-0,062 *$ & 0,029 \\
\hline \multicolumn{5}{|c|}{ Berufsstatus (Ref.: vollzeit erwerbstätig) } \\
\hline Teilzeit, geringfügig erwerbstätig & $0,068^{*}$ & 0,032 & $0,066^{*}$ & 0,032 \\
\hline Arbeitslos, -suchend & 0,064 & 0,054 & 0,051 & 0,054 \\
\hline Hausfrau/-mann & $0,103^{*}$ & 0,046 & $0,099 *$ & 0,046 \\
\hline (Früh-)Rente & $0,087 * *$ & 0,033 & $0,084 *$ & 0,033 \\
\hline Anderes & 0,021 & 0,048 & 0,017 & 0,048 \\
\hline Materielle Lage $^{\mathrm{a}}$ & $0,095 * * *$ & 0,011 & $0,091 * * *$ & 0,011 \\
\hline Wohndauer (Ref.: unter 1 Jahr) & 0,000 & - & 0,000 & - \\
\hline 1 bis 2 Jahre & 0,031 & 0,057 & 0,026 & 0,057 \\
\hline 2 bis 5 Jahre & 0,004 & 0,048 & 0,002 & 0,048 \\
\hline 5 bis 10 Jahre & 0,053 & 0,049 & 0,052 & 0,048 \\
\hline 10 bis 20 Jahre & $0,104 *$ & 0,049 & $0,103 *$ & 0,049 \\
\hline 20 Jahre und mehr & $0,136^{* *}$ & 0,048 & $0,137 * *$ & 0,048 \\
\hline Viktimisierung Eigentumsdelikte & $0,154 * * *$ & 0,019 & $0,153 * * *$ & 0,019 \\
\hline Viktimisierung Gewaltdelikte & $0,485 * * *$ & 0,025 & $0,485^{* * *}$ & 0,025 \\
\hline \multicolumn{5}{|l|}{ L2 $(N=140 \text { Stadtviertel })^{\mathrm{a}}$} \\
\hline SSO physische Unordnung & $0,281 * * *$ & 0,028 & $0,100 * * *$ & 0,025 \\
\hline SSO soziale Unordnung & $0,066^{*}$ & 0,028 & $0,043 *$ & 0,019 \\
\hline$\%$-Anteil Nicht-EU-Ausländer & - & - & $0,173 * * *$ & 0,052 \\
\hline \%-Anteil EU-Ausländer & - & - & 0,028 & 0,027 \\
\hline$\%$-Anteil Unterstützungsempfänger & - & - & $0,116^{*}$ & 0,047 \\
\hline Pol.registr. Sachbeschädigung & - & - & $0,046^{*}$ & 0,021 \\
\hline Konstante & $-0,592 * * *$ & 0,125 & $-0,551 * * *$ & 0,123 \\
\hline Varianzkomponente L1 & $0,500 * * *$ & 0,005 & $0,500 * * *$ & 0,005 \\
\hline Varianzkomponente L2 & $0,084 * * *$ & 0,006 & $0,030 * * *$ & 0,002 \\
\hline Varianzreduktion $\mathrm{L}^{\mathrm{b}}(\%)$ & 11,3 & & 11,3 & \\
\hline
\end{tabular}


Tab. 1 Lineare Mehrebenen-Regression der wahrgenommenen Unordnung im Wohngebiet (Köln und Essen) (Fortsetzung)

\begin{tabular}{|c|c|c|c|c|}
\hline & Modell & & Modell 2 & \\
\hline & $\mathrm{B}$ & SE & $\mathrm{B}$ & SE \\
\hline Varianzreduktion $\mathrm{L}^{\mathrm{c}}(\%)$ & 54,9 & & 83,9 & \\
\hline ICC (30,5\% im Nullmodell) $(\%)$ & 14,4 & & 5,7 & \\
\hline$-\mathrm{LL}$ & $-6728,7$ & & $-6669,7$ & \\
\hline
\end{tabular}

L1-Prädiktoren sind unzentriert (kategoriale Variablen) oder grand mean-zentriert (intervallskalierte Variablen). Ausgeschlossen als nicht-signifikant: Geschlecht, Familienstand, Hausbesitz, Kinder im Haushalt $+p<0,10, * p<0,05, * * p<0,01, * * * p<0,001$

${ }^{a}$ Prädiktor ist standardisiert (Mittelwert $=0$, Standardabweichung $=1$ )

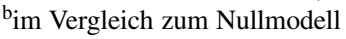

${ }^{c}$ im Vergleich zum konditionalen Modell unter Kontrolle der soziodemographischen Merkmale

zehnjähriger Wohndauer mehr Unordnung wahr als andere Befragte. Befragte mit einem nicht-europäischen (z. B. türkischen) Migrationshintergrund nehmen etwas weniger Unordnung wahr als einheimisch-deutsche. Der relativ starke Alterseffekt verläuft kurvilinear: Bis etwa 50 Jahre nehmen Befragte mehr Unordnung war, danach jedoch immer weniger. Der mit Abstand stärkste individuelle Effekt rührt von persönlichen Gewalterfahrungen her: Wer Opfer eines Gewaltdelikts wurde, nimmt erheblich mehr Unordnung war als andere (Eifler et al. 2009). Keine Bedeutung haben demgegenüber Geschlecht, Familienstand, Hausbesitz oder die Existenz von Kindern im Haushalt.

Im zweiten Modell werden zusätzlich auch soziodemographische Merkmale der Wohngebiete und die Belastung mit polizeilich registrierten Sachbeschädigungen aufgenommen. Den mit Abstand stärksten Effekt hat nunmehr der Anteil der NichtEU-Ausländer*innen (während der Anteil der EU-Ausländer*innen gar keinen Einfluss hat), gefolgt von konzentrierter Armut, während der Effekt der durch SSO gemessenen physischen Unordnung gegenüber dem vorherigen Modell um etwa zwei Drittel schrumpft. Die Aufklärung der Varianz zwischen den Wohngebieten steigt von 55 auf $84 \%$ an. Es sind also vor allem sichtbare ethnische Minderheiten, nicht ethnische Minderheiten generell, die die Wahrnehmungsverzerrung erklären können. Ebenso wie in früheren Studien aus den USA erklären die soziodemographische Struktur der Wohngebiete und insbesondere der Anteil sichtbarer ethnischer Minderheiten die Unterschiede in den subjektiven Wahrnehmungen von Unordnung wesentlich stärker als das objektive Ausmaß der Unordnung (Drakulich 2013; Sampson und Raudenbush 2004). Dieser Zusammenhang gilt (ebenso wie in Chicago) für einheimisch-deutsche wie für migrantische Befragte gleichermaßen. Dies entspricht auch den Ergebnissen der Hamburger Studie, in der allerdings nicht zwischen Armut und ethnischer Diversität unterschieden wurde (Häfele 2013).

Welche Rolle bei der Wahrnehmungsverzerrung spielen ethnische Vorurteile der Bewohner*innen? Um diese bislang noch unerforschte Frage zu beantworten, haben wir das Mehrebenenmodell um einen Interaktionseffekt (cross-level interaction) zwischen den xenophoben Einstellungen der individuellen Befragten und dem Bevölkerungsanteil der Nicht-EU Ausländer*innen erweitert (Tab. 2, Modell 3) und das Ergebnis dieser hoch signifikanten Interaktion graphisch dargestellt (Abb. 2). Hier zeigt sich zunächst, dass Bewohner*innen mit stärkeren xenophoben Einstel- 
Tab. 2 Lineare Mehrebenen-Regression der wahrgenommenen Unordnung im Wohngebiet (Köln und Essen)

\begin{tabular}{|c|c|c|}
\hline & \multicolumn{2}{|l|}{ Modell 3} \\
\hline & $\mathrm{B}$ & SE \\
\hline \multicolumn{3}{|l|}{ L1 $(N=6136$ Befragte $)$} \\
\hline Xenophobe Einstellungen ${ }^{\mathrm{a}}$ & $0,135 * * *$ & 0,010 \\
\hline \multicolumn{3}{|c|}{ Weitere L1-Prädiktoren wie in Modell $1 \& 2$} \\
\hline \multicolumn{3}{|c|}{$\mathrm{L2}^{\mathrm{a}}(N=140$ Stadtviertel) und L1/L2-Interaktion } \\
\hline SSO physische Unordnung & $0,107 * * *$ & 0,025 \\
\hline SSO soziale Unordnung & $0,048^{*}$ & 0,019 \\
\hline \%-Anteil Nicht-EU-Ausländer & $0,165^{* *}$ & 0,053 \\
\hline $\mathrm{X}$ xenophobe Einstellungen & $0,037 * * *$ & 0,009 \\
\hline \%-Anteil EU-Ausländer & 0,029 & 0,027 \\
\hline \%-Anteil Unterstützungsempfänger & $0,097^{*}$ & 0,048 \\
\hline Pol. registr. Sachbeschädigungen & $0,054^{*}$ & 0,021 \\
\hline Konstante & $-0,573 * * *$ & 0,121 \\
\hline Varianzkomponente L1 & $0,483^{* * *}$ & 0,004 \\
\hline Varianzkomponente L2 & $0,031 * * *$ & 0,003 \\
\hline Varianzreduktion $\mathrm{L} 1^{\mathrm{b}}(\%)$ & 14,4 & \\
\hline Varianzreduktion $\mathrm{L} 2^{\mathrm{c}}(\%)$ & 83,3 & \\
\hline ICC $(30,5 \%$ im Nullmodell) $(\%)$ & 6,1 & \\
\hline$-L L$ & $-6566,8$ & \\
\hline
\end{tabular}

Anmerkungen siehe Tab. 1

lungen in allen Arten von Wohngebieten mehr Unordnung wahrnehmen. Entscheidend für unsere Frage ist aber, dass der Effekt des Bevölkerungsanteils von NichtEU Ausländer*innen auf die Wahrnehmungsverzerrung der Bewohner*innen umso schwächer wird, je geringer deren xenophobe Einstellungen sind. Bewohner*innen mit besonders starken xenophoben Einstellungen in Wohngebieten mit einem hohen Anteil sichtbarer Minderheiten nehmen relativ zur objektiven Situation am meisten Unordnung war. Für Bewohner*innen mit besonders positiven Einstellungen gegenüber Migrant*innen ist die Wahrnehmungsverzerrung sehr klein, aber nur etwa $6 \%$ der Befragten am untersten Ende der Skala für xenophobe Einstellungen zeigen sich vollständig immun. Dies bestätigt die Annahme der sozialpsychologischen Forschung, dass auch Personen ohne explizite ethnische Vorurteile dennoch implizite Vorurteile haben können, die sich auf ihre Wahrnehmungen auswirken (Dovidio et al. 2002).

\section{Ergebnisse II: Einflussfaktoren der Kriminalitätsfurcht}

Im zweiten Schritt untersuchen wir den Einfluss der durch SSO gemessenen Unordnung auf die wohngebietsbezogene Unsicherheitswahrnehmung der Bewohner*innen - einer Kernannahme des „Broken Windows“-Ansatzes - im Vergleich zu anderen sozialräumlichen Effekten und kontrastieren das Ergebnis anschließend mit dem Einfluss der subjektiv wahrgenommenen Unordnung. Während bei den 
Abb. 2 Interaktionseffekt zwischen \%-Anteil Nicht-EU Ausländer*innen und individuellen xenophoben Einstellungen auf die wahrgenommene Unordnung (Modell 3 in Tab. 2)

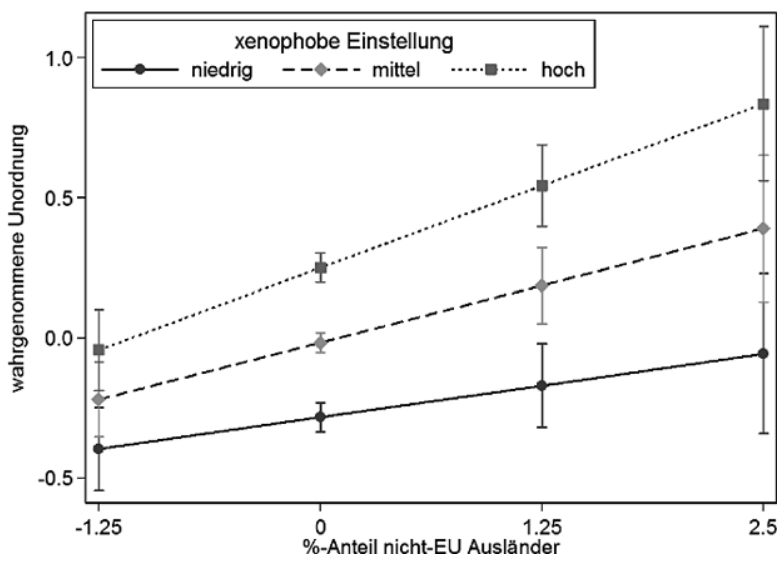

Analysen zur Wahrnehmungsverzerrung keine bedeutsamen Unterschiede zwischen den beiden Städten auftraten, stellen sich die Zusammenhänge in Köln und Essen nun deutlich anders da, weshalb wir die folgenden linearen Mehrebenenmodelle für beide Städte getrennt gerechnet haben (Tab. 3 und 4).

Die individuellen (L1-) Effekte unterscheiden sich zwischen beiden Städten jedoch kaum: Frauen, materiell schlechter Gestellte, Kriminalitätsopfer und Befragte mit xenophoben Einstellungen berichten innerhalb derselben Wohngebiete stärkere Unsicherheitsgefühle. Der Alterseffekt ist wiederum kurvilinear mit einem deutlich stärkeren Anstieg der Unsicherheit im höheren Alter. Nur in Köln berichten Verheiratete etwas stärkere und nur in Essen Befragte mit einem nicht-europäischen Migrationshintergrund etwas geringere Unsicherheitsgefühle.

Wesentliche Unterschiede zwischen den Städten finden sich bei den sozialräumlichen (L2-) Effekten: Während die durch SSO gemessene physische und soziale Unordnung unter Kontrolle sozialstruktureller Merkmale und Kriminalität in Köln gar keinen Effekt auf das Unsicherheitsgefühl hat, bestehen in Essen signifikante furchtsteigernde Einflüsse fort (Tab. 3 und 4, Modell 1). ${ }^{5}$ Dies muss als teilweise Bestätigung der „Broken Windows“-Theorie bewertet werden. Jedoch dominiert in beiden Städten klar der Effekt der sichtbaren Minderheiten als stärkster Prädiktor. Daneben wirkt sich in Köln auch die Häufigkeit von Wohnungseinbrüchen auf das Unsicherheitsgefühl aus. Köln hatte 2013 und 2014 die bundesweit zweithöchste Häufigkeitsziffer der Großstädte bei Wohnungseinbruchsdiebstahl. Dieser Effekt der Wohnungseinbrüche auf das Unsicherheitsgefühl sollte weiter untersucht werden. Die Häufigkeit von Gewaltkriminalität ebenso wie die konzentrierte Armut hat in keiner der beiden Städte einen spürbaren Effekt.

Das Modell 1 erklärt in Köln $86 \%$ und in Essen fast $90 \%$ der Varianz der Unsicherheitswahrnehmungen zwischen den Wohngebieten (ein Modell ausschließlich

\footnotetext{
5 Bei einem schrittweisen Modellaufbau in Köln wird der Koeffizient der durch SSO gemessenen physischen Unordnung unter Kontrolle der soziodemographischen Struktur des Stadtviertels, aber ohne Kontrolle der Kriminalitätsbelastung, sogar negativ; d. h. höhere Unordnung trägt zu schwächeren Unsicherheitsgefühlen bei.
} 
Tab. 3 Lineare Mehrebenen-Regression der Unsicherheitsgefühle im Wohngebiet (Köln)

\begin{tabular}{|c|c|c|c|c|c|c|}
\hline & \multicolumn{2}{|l|}{ Modell 1} & \multicolumn{2}{|l|}{ Modell 2} & \multicolumn{2}{|l|}{ Modell 3} \\
\hline & $\mathrm{B}$ & SE & $\mathrm{B}$ & SE & $\mathrm{B}$ & SE \\
\hline \multicolumn{7}{|l|}{ L1 ( $N=3859$ Befragte $)$} \\
\hline Geschlecht (Ref.: männlich) & $0,180 * * *$ & 0,018 & $0,180 * * *$ & 0,018 & $0,173 * * *$ & 0,017 \\
\hline Alter (in Dekaden) & $-0,110 * *$ & 0,041 & $-0,112 * *$ & 0,041 & $-0,125 * *$ & 0,040 \\
\hline Alter quadriert & $0,014 * * *$ & 0,004 & $0,014 * * *$ & 0,004 & $0,015 * * *$ & 0,003 \\
\hline \multicolumn{7}{|l|}{ Familienstand (Ref.: ledig) } \\
\hline Verheiratet & $0,063^{*}$ & 0,027 & $0,064 *$ & 0,027 & $0,062 *$ & 0,026 \\
\hline Geschieden & 0,029 & 0,037 & 0,031 & 0,037 & 0,033 & 0,036 \\
\hline Verwitwet & 0,055 & 0,040 & 0,056 & 0,040 & $0,079 *$ & 0,039 \\
\hline \multicolumn{7}{|c|}{ Schulbildung (Ref.: mittlere Reife) } \\
\hline Kein Abschluss & $0,187 * * *$ & 0,055 & $0,191 * * *$ & 0,055 & $0,141 * *$ & 0,053 \\
\hline Volks-/Hauptschule & $0,071 * *$ & 0,026 & $0,069 * *$ & 0,026 & $0,049 *$ & 0,025 \\
\hline (Fach-)Abitur & $-0,026$ & 0,031 & $-0,029$ & 0,031 & $-0,023$ & 0,030 \\
\hline (Fach-)Hochschule & $-0,086^{* *}$ & 0,027 & $-0,093 * * *$ & 0,027 & $-0,085^{* *}$ & 0,026 \\
\hline Materielle Lage $^{\mathrm{a}}$ & $0,055^{* * *}$ & 0,010 & $0,055^{* * *}$ & 0,010 & $0,039 * * *$ & 0,009 \\
\hline $\begin{array}{l}\text { Viktimisierung Eigentumsde- } \\
\text { likte }\end{array}$ & $0,067 * * *$ & 0,018 & $0,066 * * *$ & 0,018 & $0,040 *$ & 0,017 \\
\hline Viktimisierung Gewaltdelikte & $0,222 * * *$ & 0,023 & $0,222 * * *$ & 0,023 & $0,131 * * *$ & 0,023 \\
\hline Xenophobe Einstellung ${ }^{\mathrm{a}}$ & $0,118 * * *$ & 0,009 & $0,121 * * *$ & 0,009 & $0,088 * * *$ & 0,009 \\
\hline $\begin{array}{l}\text { Wahrgenommene } \\
\text { Unordnung }^{\mathrm{a}}\end{array}$ & - & - & - & - & $0,206 * * *$ & 0,011 \\
\hline \multicolumn{7}{|c|}{ L2 $^{\text {a }}$ ( $N=85$ Stadtviertel) und L1/L2-Interaktion } \\
\hline SSO physische Unordnung & n. s. & - & n. s. & - & n. s. & - \\
\hline SSO soziale Unordnung & n. s. & - & n. s. & - & n. s. & - \\
\hline $\begin{array}{l}\text { \%-Anteil Nicht-EU-Auslän- } \\
\text { der }\end{array}$ & $0,176^{* * *}$ & 0,014 & $0,175^{* * *}$ & 0,014 & $0,100 * * *$ & 0,012 \\
\hline X xenoph. Einstellungen & - & - & $0,034 * * *$ & 0,010 & - & - \\
\hline \%-Anteil EU-Ausländer & n. s. & - & n. s. & - & n. s. & - \\
\hline $\begin{array}{l}\text { \%-Anteil Unterstützungs- } \\
\text { empfänger }\end{array}$ & n. s. & - & n. s. & - & n. s. & - \\
\hline $\begin{array}{l}\text { Pol. registr. Wohnungsein- } \\
\text { brüche }\end{array}$ & $0,063 * * *$ & 0,012 & $0,063 * * *$ & 0,012 & $0,067 * * *$ & 0,010 \\
\hline Pol. registr. Gewaltdelikte & n. s. & - & n.s. & - & n. s. & - \\
\hline Konstante & $0,421 * *$ & 0,135 & $0,431 * *$ & 0,135 & $0,465 * * *$ & 0,124 \\
\hline Varianzkomponente L1 & $0,286 * * *$ & 0,003 & $0,285 * * *$ & 0,003 & $0,266^{* * *}$ & 0,003 \\
\hline Varianzkomponente L2 & $0,004 * * *$ & 0,001 & $0,005 * * *$ & 0,001 & $0,001 * * *$ & 0,001 \\
\hline Varianzreduktion $\mathrm{L}^{\mathrm{b}}(\%)$ & 17,1 & & 17,4 & & 22,9 & \\
\hline Varianzreduktion L2 $2^{\mathrm{c}}(\%)$ & 86,2 & & 85,5 & & 95,4 & \\
\hline $\begin{array}{l}\text { ICC (14,8\% im Nullmodell) } \\
(\%)\end{array}$ & 1,5 & & 1,6 & & 0,5 & \\
\hline
\end{tabular}


Tab. 3 Lineare Mehrebenen-Regression der Unsicherheitsgefühle im Wohngebiet (Köln) (Fortsetzung)

\begin{tabular}{|c|c|c|c|c|c|c|}
\hline & Modell 1 & & Modell 2 & & Modell 3 & \\
\hline & B & SE & B & SE & B & SE \\
\hline$\overline{-L L}$ & $-3080,3$ & & $-3074,2$ & & $-2927,4$ & \\
\hline
\end{tabular}

L1-Prädiktoren sind unzentriert (kategoriale Variablen) oder grand mean-zentriert (intervallskalierte Variablen). Ausgeschlossen als nicht-signifikant: Migrationshintergrund, Berufsstatus, Kinder im Haushalt, Wohndauer

$+p<0,10, * p<0,05, * * p<0,01, * * * p<0,001$

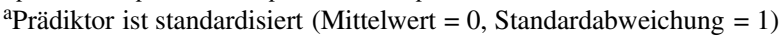

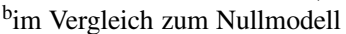

cim Vergleich zum konditionalen Modell unter Kontrolle der soziodemographischen Merkmale

mit der durch SSO gemessenen physischen und sozialen Unordnung erklärt in Köln nur $10 \%$, in Essen aber bereits $65 \%$ der Varianz auf der Stadtviertelebene).

Im zweiten Modell untersuchen wir wiederum die Interaktion zwischen der sozialräumlichen Konzentration sichtbarer Minderheiten und den individuellen xenophoben Einstellungen. Wie bei der Wahrnehmungsverzerrung auch gilt beim Unsicherheitsempfinden, dass der furchtsteigernde Effekt des Anteils sichtbarer Minderheiten im Wohngebiet sehr stark von den individuellen Einstellungen der Bewohner*innen zur Migration abhängig ist. Wir illustrieren diese Wechselwirkung für Essen, wo sie noch ausgeprägter ist als in Köln, in Abb. 3: Befragte mit sehr positiven Einstellungen zu Migration fühlen sich selbst dann nicht unsicherer, wenn sie in Wohngebieten mit einem hohen Anteil von Nicht-EU-Ausländer*innen wohnen. Andererseits fühlen sich Befragte mit ausgeprägten xenophoben Einstellungen in Wohngebieten mit einem hohen Anteil sichtbarer Minderheiten sehr unsicher.

Schließlich testen wir im dritten Modell, wie stark im Vergleich zu Modell 1 der Effekt der subjektiv wahrgenommenen Unordnung auf das Unsicherheitsempfinden ist und wie sich dadurch die Einflussstärken der übrigen Prädiktoren verändern. Wie in der Einleitung erwähnt konnte die Mehrzahl der bisherigen Studien ausschließlich diese subjektiven Wahrnehmungen der Befragten verwenden. Erwartungsgemäß hat die subjektiv wahrgenommene Unordnung einen extrem starken Effekt auf das Unsicherheitsempfinden, erhöht den Anteil der erklärten Varianz auf L1 und L2 und vermittelt einen erheblichen Teil der Effekte sowohl der systematisch beobachteten Unordnung (in Essen) als auch der Konzentration sichtbarer Minderheiten (in Köln und Essen), deren Koeffizienten um rund $45 \%$ sinken. Demgegenüber bleibt in Köln der Koeffizient der Wohnungseinbrüche gleich stark. Auch die individuellen Effekte der materiellen Lage und der Opfererfahrungen werden teilweise über die wahrgenommene Unordnung vermittelt. Wir interpretieren dieses Ergebnis als Beleg für die enge Verknüpfung der subjektiven Wahrnehmungen von Unordnung und Unsicherheit, die in der bisherigen Forschung zu einer Überschätzung der Konsequenzen urbaner Unordnung geführt hat. Unsere Modelle legen den Schluss nahe, dass die subjektive Wahrnehmung von Unordnung eine wesentliche Vermittlungsrolle bei der Entwicklung von urbanen Unsicherheitsgefühlen in sozial benachteiligten Wohngebieten hat. 
Tab. 4 Lineare Mehrebenen-Regression der Unsicherheitsgefühle im Wohngebiet (Essen)

\begin{tabular}{|c|c|c|c|c|c|c|}
\hline & \multicolumn{2}{|l|}{ Modell 1} & \multicolumn{2}{|l|}{ Modell 2} & \multicolumn{2}{|l|}{ Modell 3} \\
\hline & $\mathrm{B}$ & SE & $\mathrm{B}$ & SE & $\mathrm{B}$ & SE \\
\hline \multicolumn{7}{|l|}{ L1 ( $N=2268$ Befragte $)$} \\
\hline Geschlecht (Ref.: männlich) & $0,207 * * *$ & 0,023 & $0,202 * * *$ & 0,023 & $0,207 * * *$ & 0,022 \\
\hline Alter (in Dekaden) & $-0,121 *$ & 0,049 & $-0,123^{*}$ & 0,048 & $-0,159 * * *$ & 0,047 \\
\hline Alter quadriert & $0,015^{* * *}$ & 0,004 & $0,016 * * *$ & 0,004 & $0,019 * * *$ & 0,004 \\
\hline \multicolumn{7}{|l|}{ Migrationshintergrund (Ref. nein) } \\
\hline Europäische Herkunft & $-0,056$ & 0,049 & $-0,049$ & 0,048 & $-0,054$ & 0,047 \\
\hline Nicht-europäische Herkunft & $-0,118^{*}$ & 0,046 & $-0,099 *$ & 0,046 & $-0,098 *$ & 0,045 \\
\hline \multicolumn{7}{|l|}{ Schulbildung (Ref.: Mittlere Reife) } \\
\hline Kein Abschluss & 0,107 & 0,076 & 0,108 & 0,076 & 0,089 & 0,073 \\
\hline Volks-/Hauptschule & 0,024 & 0,032 & 0,020 & 0,032 & 0,023 & 0,031 \\
\hline (Fach-)Abitur & $-0,029$ & 0,041 & $-0,031$ & 0,041 & $-0,043$ & 0,040 \\
\hline (Fach-)Hochschule & $-0,083 *$ & 0,036 & $-0,096^{* *}$ & 0,036 & $-0,079 *$ & 0,035 \\
\hline Materielle Lage $^{\mathrm{a}}$ & $0,062 * * *$ & 0,012 & $0,063 * * *$ & 0,012 & $0,045^{* * *}$ & 0,012 \\
\hline $\begin{array}{l}\text { Viktimisierung Eigentumsde- } \\
\text { likte }\end{array}$ & $0,118 * * *$ & 0,023 & $0,119 * * *$ & 0,023 & $0,091 * * *$ & 0,023 \\
\hline Viktimisierung Gewaltdelikte & $0,238 * * *$ & 0,031 & $0,229 * * *$ & 0,031 & $0,141 * * *$ & 0,031 \\
\hline Xenophobe Einstellung ${ }^{\mathrm{a}}$ & $0,140 * * *$ & 0,012 & $0,129 * * *$ & 0,013 & $0,119 * * *$ & 0,012 \\
\hline Wahrgenommene Unordnung ${ }^{a}$ & - & - & - & - & $0,196 * * *$ & 0,015 \\
\hline \multicolumn{7}{|c|}{ L2 $^{\mathrm{a}}(N=55$ Stadtviertel) und L1/L2-Interaktion } \\
\hline SSO physische Unordnung & $0,050 * *$ & 0,018 & $0,050 * *$ & 0,018 & $0,029 *$ & 0,015 \\
\hline SSO soziale Unordnung & $0,055^{* *}$ & 0,020 & $0,056^{* *}$ & 0,020 & $0,044 * *$ & 0,017 \\
\hline$\%$-Anteil Nicht-EU-Ausländer & $0,149 * * *$ & 0,020 & $0,137 * * *$ & 0,020 & $0,085^{* * *}$ & 0,017 \\
\hline $\mathrm{X}$ xenoph. Einstellungen & - & - & $0,049 * * *$ & 0,010 & - & - \\
\hline \%-Anteil EU-Ausländer & n.s. & - & n. s. & - & n. s. & - \\
\hline $\begin{array}{l}\% \text {-Anteil Unterstützungsemp- } \\
\text { fänger }\end{array}$ & n. s. & - & n. s. & - & n. s. & - \\
\hline $\begin{array}{l}\text { Pol. registr. Wohnungseinbrü- } \\
\text { che }\end{array}$ & n. s. & - & n. s. & - & n. s. & - \\
\hline Pol. registr. Gewaltdelikte & n. s. & - & n. s. & - & n. s. & - \\
\hline Konstante & $0,876^{* * *}$ & 0,141 & $0,885^{* * * *}$ & 0,141 & $1,032 * * *$ & 0,137 \\
\hline Varianzkomponente L1 & $0,277 * * *$ & 0,004 & $0,274 * * *$ & 0,004 & $0,261 * * *$ & 0,004 \\
\hline Varianzkomponente L2 & $0,006 * * *$ & 0,001 & $0,006 * * *$ & 0,001 & $0,002 * * *$ & 0,001 \\
\hline Varianzreduktion $\mathrm{L}^{\mathrm{b}}(\%)$ & 19,5 & & 20,2 & & 24,1 & \\
\hline Varianzreduktion $\mathrm{L} 2^{\mathrm{c}}(\%)$ & 88,9 & & 89,2 & & 96,1 & \\
\hline $\begin{array}{l}\text { ICC }(19,9 \% \text { im Nullmodell }) \\
(\%)\end{array}$ & 2,1 & & 1,6 & & 0,8 & \\
\hline$-\mathrm{LL}$ & $-1778,4$ & & $-1767,4$ & & $-1701,3$ & \\
\hline
\end{tabular}

L1-Prädiktoren sind unzentriert (kategoriale Variablen) oder grand mean-zentriert (intervallskalierte Variablen). Ausgeschlossen als nicht-signifikant: Berufsstatus, Kinder im Haushalt, Wohndauer $+p<0,10, * p<0,05, * * p<0,01, * * * p<0,001$

aPrädiktor ist standardisiert (Mittelwert $=0$, Standardabweichung $=1$ )

bim Vergleich zum Nullmodell

cim Vergleich zum konditionalen Modell unter Kontrolle der soziodemographischen Merkmale 
Abb. 3 Interaktionseffekt zwischen \%-Anteil Nicht-EU Ausländer*innen und individuellen xenophoben Einstellungen auf das Unsicherheitsgefühl im Wohngebiet (Essen, Modell 2 in Tab. 4)

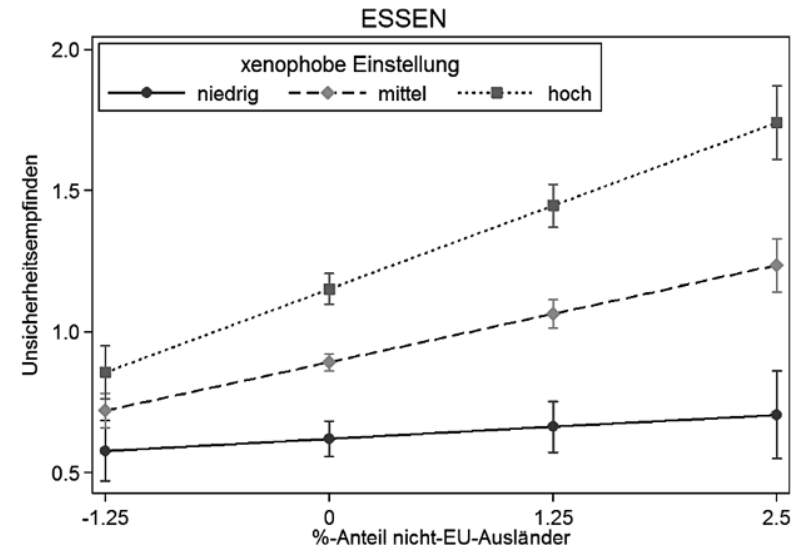

\section{Zusammenfassung und Diskussion}

Kurzgefasst sprechen die empirischen Ergebnisse gegen eine maßgebliche Rolle urbaner Unordnung bei der Entstehung lokaler Unsicherheitswahrnehmungen und lenken die Aufmerksamkeit stattdessen auf die ethnische Diversität in Wohngebieten als dem mit Abstand stärksten sozialräumlichen Einfluss. Jedoch sprechen die unterschiedlichen Ergebnisse in Köln und Essen gegen eine pauschale Ablehnung des „Broken Windows“-Ansatzes (siehe dazu unten).

Bereits in früheren Studien zeigte sich, dass in erster Linie eine problematische Sozialstruktur in segregierten Wohngebieten bei den Menschen Unsicherheit auslöste (Lüdemann 2006; Oberwittler 2008). Auch wenn ethnische und soziale Segregation sehr eng miteinander verknüpft sind (die Korrelation zwischen den beiden Prädiktoren beträgt in Köln $r=0,74$ und in Essen $r=0,91$ ), erweist sich der Anteil sichtbarer Minderheiten gegenüber der konzentrierten Armut dennoch als dominanter Einfluss. ${ }^{6}$ Dieses Ergebnis unterstützt die breite Forschung zu den negativen Auswirkungen ethnischer Diversiät auf das kollektive Sozialkapital von Wohngebieten, die sich mit ganz wenigen Ausnahmen (v. a. London) in den meisten untersuchten Städten gezeigt haben (van der Meer und Tolsma 2014). ${ }^{7}$ Diese übereinstimmenden Befunde stellen eine große Herausforderung an die Sozialpolitik und an die Entwicklung großstädtischer Wohnquartiere dar, zumal in Zeiten verstärkter Einwanderung aus außereuropäischen Regionen (El-Mafaalani et al. 2015).

Jedoch zeigen die empirischen Ergebnisse auch, dass sozialräumliche Merkmale nicht unmittelbar und unbedingt Unsicherheit erzeugen, sondern erst durch subjektiv unterschiedlich geprägte „Wahrnehmungsfilter“. In unseren Mehrebenenmodellen wurden die Effekte der sozialräumlichen Merkmale maßgeblich durch die individuellen Einstellungen zu Migration moderiert: Eine positive Einstellung zu

\footnotetext{
${ }^{6}$ Wegen potenzieller Multikollinearität haben wir alternative Modelle mit jeweils nur einem der beiden Variablen gerechnet. Der Anteil ausländischer Bewohner*innen hat auch dann eine höhere Erklärkraft als der Anteil der Unterstützungsempfänger*innen.
}

7 Vgl. dazu den Beitrag von Birte Gundelach zum vorliegenden Heft. 
Migration schwächt die furchtsteigernden Effekte ethnischer Diversität deutlich ab, während ablehnende und xenophobe Einstellungen sie verstärken. Dies unterstreicht die schon lange bekannte Verknüpfung des Begriffspaars „Kriminalität“ und „Ausländer" in der Wahrnehmung vieler Menschen (Sessar 2010). Da hinter der Einstellung zu Migration weitere und grundlegendere sozialpsychologische Dimensionen vermutet werden können, sind Forschungsansätze lohnend, die die Wechselwirkungen zwischen sozialräumlichen Bedingungen und individuellen Persönlichkeits- und Einstellungsmustern untersuchen. So wurde beispielsweise ein stärkeres Bedürfnis nach kognitiver Geschlossenheit bzw. eine geringere Ambiguitätstoleranz mit gesteigerter Kriminalitätsfurcht und einer stärker ausgeprägten Abgrenzung gegenüber Fremden in Verbindung gebracht (Kruglanski et al. 2006; Jackson 2015; vgl. Jost et al. 2003; Obergfell-Fuchs und Kury 1996).

Unsere Ergebnisse unterstreichen außerdem die enge Verknüpfung von Unsicherheitsempfinden und subjektiv wahrgenommener Unordnung, die in vielen früheren Studien fälschlicherweise als Beleg für die furchterzeugenden Wirkungen von urbaner Unordnung bewertet wurden. Durch den Vergleich von systematisch beobachteter und subjektiv wahrgenommener Unordnung konnten wir zeigen, dass auch die Wahrnehmung von Unordnung - ebenso wie die Unsicherheitswahrnehmung - in erster Linie vom Bevölkerungsanteil sichtbarer Minderheiten im Wohngebiet und zusätzlich auch von konzentrierter Armut angetrieben wird. Relativ zum „objektiven“ Ausmaß der Unordnung, ,sehen“ Bewohner*innen dort mehr Unordnung, wo die ethnische Diversität hoch ist. Die bisherigen Erkenntnisse zu dieser „Wahrnehmungsverzerrung“ (Drakulich 2013; Häfele 2013; Sampson und Raudenbush 2004) haben wir um den Befund ergänzt, dass dieser sozialräumliche Effekt (wiederum parallel zum Unsicherheitsempfinden) von der individuellen Einstellung zu Migration abhängt. Explizite xenophobe Einstellungen verstärken die Wahrnehmungsverzerrung deutlich, aber auch implizite ethnische Vorurteile (die in unserer Studie nicht gemessen wurden) unterstützen vermutlich Tendenzen zur Wahrnehmungsverzerrung (Drakulich 2015). Insgesamt unterstützen die Ergebnisse die Interpretation von Unsicherheit und Unordnung als verwandte Kognitionen sozialräumlicher Problemlagen, die bei vielen Bewohner*innen Unbehagen auslösen und sich am Thema Kriminalität kristallisieren (Hirtenlehner und Farrall 2013; Sampson 2009).

Weniger verallgemeinerungsfähig sind die Ergebnisse hinsichtlich der „Broken Windows"-These, dass Unordnung im öffentlichen Raum die Unsicherheitsgefühle steigert. Diese Hypothese wird in Essen partiell bestätigt und in Köln klar widerlegt. In Essen erklärt die systematisch beobachtete Unordnung alleine $65 \%$ der Varianz der Stadtviertelunterschiede in den Unsicherheitsgefühlen, in Köln dagegen nur $10 \%$. Dieses unerwartete Ergebnis fordert zum Nachdenken auf: Was unterscheidet die beiden Städte und ihre Bewohner*innen so voneinander, dass die Zeichen urbaner Unordnung wie Müll und Graffiti offensichtlich so unterschiedlich bewertet werden? Eine erste explorative Betrachtung deutet darauf hin, dass in Köln einige innenstadtnahe Gebiete mit einer lebendigen urbanen Infrastruktur zwar objektiv viele Zeichen von Unordnung aufweisen, diese aber von den Bewohner*innen nicht als bedrohlich wahrgenommen werden. Möglicherweise wird Unordnung in diesem Kontext als Begleiterscheinung eines vielfältigen urbanen (Nacht-)Lebens toleriert, während dieselbe Unordnung in anderen Kontexten zu den Zumutungen eines im 
Abstieg befindlichen ,sozialen Brennpunktes“ gezählt wird. Ob diese und andere kontextabhängige Faktoren die Unterschiede in den Wahrnehmungsfiltern urbaner Unordnung erklären können, bleibt weiteren Analysen vorbehalten.

Funding Open access funding provided by Max Planck Society.

Open Access Dieser Artikel wird unter der Creative Commons Namensnennung 4.0 International Lizenz (http://creativecommons.org/licenses/by/4.0/deed.de) veröffentlicht, welche die Nutzung, Vervielfältigung, Bearbeitung, Verbreitung und Wiedergabe in jeglichem Medium und Format erlaubt, sofern Sie den/die ursprünglichen Autor(en) und die Quelle ordnungsgemäß nennen, einen Link zur Creative Commons Lizenz beifügen und angeben, ob Änderungen vorgenommen wurden.

\section{Anhang}

Tab. 5 Deskriptive Statistik

\begin{tabular}{ll}
\hline & $\%$ \\
\hline Geschlecht: weiblich & 54,8 \\
Migrationshintergrund & \\
Einheimisch-deutsch & 80,4 \\
Europäische Herkunft & 7,0 \\
Nicht-europäische Herkunft & 10,4 \\
K. A. & 2,1 \\
Schulbildung & \\
Kein Abschluss & 2,8 \\
Volks-/Hauptschule & 33,8 \\
Mittlere Reife & 19,6 \\
(Fach-)Abitur & 12,9 \\
(Fach-)Hochschule & 23,7 \\
K. A. & 7,1 \\
Erwerbstatus & \\
Vollzeit erwerbstätig & 26,6 \\
Teilz/geringfügig erwerbstätig & 11,8 \\
Arbeitlos, -suchend & 3,6 \\
Hausfrau/-mann & 5,1 \\
(Früh-)Rente & 46,0 \\
Anderes & 4,3 \\
K. A. & 2,6 \\
Wohnstatus & \\
Miete & 58,1 \\
Eigentum & 39,9 \\
Heimbewohner, k. A. & 1,9 \\
Familienstand & \\
Ledig & 16,2 \\
Verheiratet & 61,5 \\
Geschieden & 9,8 \\
Verwitwet & 10,7 \\
\hline & \\
\hline & \\
\hline
\end{tabular}


Tab. 5 Deskriptive Statistik (Fortsetzung)

\begin{tabular}{ll}
\hline & $\%$ \\
\hline K. A. & 1,9 \\
Wohndauer & \\
Bis 1 Jahr & 4,8 \\
1 bis 2 Jahre & 5,4 \\
2 bis 5 Jahre & 14,0 \\
5 bis 10 Jahre & 16,0 \\
10 bis 20 Jahre & 18,7 \\
20 J. oder mehr & 40,6 \\
K. A. & 0,5 \\
Kinder im Haushalt: ja & 23,5 \\
Viktimisierung Eigentumsdelikte; ja & 48,0 \\
Viktimisierung Gewaltdelikte; ja & 18,2 \\
\hline
\end{tabular}

$N=6136$ Befragte in Köln und Essen

Tab. 6 Deskriptive Statistik (intervallskalierte Variablen)

\begin{tabular}{|c|c|c|c|c|}
\hline & Mittelwert & St.abw & Min & Max \\
\hline \multicolumn{5}{|l|}{ Befragte $(\mathrm{L1}, N=6136)$} \\
\hline Alter (in Dekaden) & 6,0 & 1,6 & 2,5 & 9,0 \\
\hline Wahrgenommene Unordnung & 0,0 & 1,0 & $-2,0$ & 3,0 \\
\hline Materielle Lage & 0,0 & 1,0 & $-1,2$ & 2,9 \\
\hline \multicolumn{5}{|l|}{ Stadtviertel $(\mathbf{L 2}, N=140)$} \\
\hline \multicolumn{5}{|c|}{ Alle Variablen sind in den Regressionsmodellen standardisiert } \\
\hline $\mathrm{SSO}^{\mathrm{a}}$ physische Unordnung (log.) & 3,5 & 0,7 & 1,5 & 4,7 \\
\hline $\mathrm{SSO}^{\mathrm{a}}$ soziale Unordnung (log.) & 0,2 & 0,2 & 0,0 & 1,6 \\
\hline \%-Anteil Nicht-EU-Ausländer & 13,9 & 10,5 & 0,7 & 44,4 \\
\hline \%-Anteil EU-Ausländer & 6,9 & 3,6 & 1,9 & 25,5 \\
\hline \%-Anteil Unterstützungsempfänger SGB II/XII & 18,0 & 13,0 & 0,8 & 56,9 \\
\hline Polizeilich registrierte Sachbeschädigungen (log.) & 6,6 & 0,6 & 4,2 & 8,4 \\
\hline Polizeilich registrierte Wohnungseinbrüche (log.) & 2,5 & 0,4 & 1,5 & 3,7 \\
\hline Polizeilich registrierte Gewaltdelikte (log.) & 7,0 & 0,9 & 4,6 & 10,1 \\
\hline
\end{tabular}

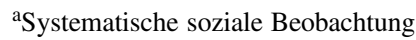




\section{Literatur}

Albrecht, Hans-Jörg. 2002. Immigration, crime and unsafety. In Crime and insecurity. The governance of safety in Europe, Hrsg. A. Crawford, 159-185. Cullompton: Willan.

Brunton-Smith, Ian, und Jonathan Jackson. 2012. Urban fear and its roots in place. In Urban fabric of crime and fear, Hrsg. V. Ceccato, 55-84. New York, Dordrecht, Heidelberg, and London: Springer.

Brunton-Smith, Ian, und Patrick Sturgis. 2011. Do neighborhoods generate fear of crime? An empirical test using the british crime survey. Criminology 49(2):331-369.

Brunton-Smith, Ian, Jonathan Jackson, und Alex Sutherland. 2014. Bridging structure and perception: on the neighbourhood ecology of beliefs and worries about violent crime. British Journal of Criminology 54(4):503-526.

Chiricos, Ted, Ranee McEntire, und Marc Gertz. 2001. Perceived racial and ethnic composition of neighborhood and percieved risk of crime. Social Problems 48(3):322-340.

Correll, Joshua, Bernadette Park, Charles M. Judd, und Bernd Wittenbrink. 2002. The police officer's dilemma: using ethnicity to disambiguate potentially threatening individuals. Journal of Personality and Social Psychology 83(6):1314-1329.

Covington, Jeanette, und Ralph B. Taylor. 1991. Fear of crime in urban residential neighborhoods: implications of between and within-neighborhood sources for current models. The Sociological Quarterly 32(2):231-249.

Dovidio, John F., Kerry Kawakami, und Samuel L. Gaertner. 2002. Implicit and explicit prejudice and interracial interaction. Journal of Personality and Social Psychology 82(1):62-68.

Dovidio, John F., Samuel L. Gaertner, und Adam R. Pearson. 2016. Racism among the well intentioned: bias without awareness. In The psychology of good and evil, Hrsg. A. Miller, 377-393. New York: Guildford Press.

Drakulich, Kevin M. 2013. Perceptions of the local danger posed by crime: race, disorder, informal control, and the police. Social Science Research 42(3):611-632.

Drakulich, Kevin M. 2015. Explicit and hidden racial bias in the framing of social problems. Social Problems 62(3):391-418.

Eifler, Stefanie, Daniela Thume, und Rainer Schnell. 2009. Unterschiede zwischen subjektiven und objektiven Messungen von Zeichen öffentlicher Unordnung (,Signs of incivility“). In Umfrageforschung. Herausforderungen und Grenzen, Hrsg. M. Weichbold, J. Bacher, und C. Wolf, 415-442. Wiesbaden: VS.

El-Mafaalani, Aladin, Sebastian Kurtenbach, und Klaus Peter Strohmeier (Hrsg.). 2015. „Auf die Adresse kommt es an... “ - Segregierte Stadtteile als Problem- und Möglichkeitsräume begreifen. Weinheim: Beltz.

Erikson, Kai T. 1966. Wayward puritans. A study in the sociology of deviance. New York: Wiley.

Farrall, Stephen, Jonathan Jackson, und Emily Gray. 2009. Social order and the fear of crime in contemporary times. Oxford: Oxford University Press.

Fitzgerald, Jennifer, K. Amber Curtis, und Catherine L. Corliss. 2012. Anxious publics: worries about crime and immigration. Comparative Political Studies 45(4):477-506.

Franzini, Luisa, Margaret O’Brien Caughy, Saundra Murray Nettles, und Patricia O'Campo. 2008. Perceptions of disorder: contributions of neighborhood characteristics to subjective perceptions of disorder. Journal of Environmental Psychology 28(1):83-93.

Golden, Kristin M. 2012. The effect of static, dynamic, and perceptual measures of minority threat on fear of crime. Journal of Ethnicity in Criminal Justice 10(2):108-128.

Häfele, Joachim. 2013. Die Stadt, das Fremde und die Furcht vor Kriminalität. Eine Mehrebenenanalyse zum Einfluss von urbanen Disorder-Phänomenen auf personale Kriminalitätseinstellungen. Wiesbaden: Springer VS.

Hale, Chris. 1996. Fear of crime: a review of the literature. International Review of Victimology 4(2):79-150.

Harcourt, Bernard E. 2005. Illusion of order. The false promise of broken windows policing. Cambridge: Harvard University Press.

Hipp, John R. 2010. Resident perceptions of crime and disorder: How much is ,bias“, and how much is social environment differences? Criminology 48(2):475-508.

Hirtenlehner, Helmut. 2006. Kriminalitätsfurcht - Ausdruck generalisierter Ängste oder schwindender Gewissheiten? Kölner Zeitschrift für Soziologie und Sozialpsychologie 58(2):307-331.

Hirtenlehner, Helmut, und Stephen Farrall. 2013. Anxieties about modernization, concerns about community, and fear of crime: testing two related models. International Criminal Justice Review 23(1):5-24. 
Hirtenlehner, Helmut, und Dina Hummelsheim. 2015. Kriminalitätsfurcht und Sicherheitsempfinden: Die Angst der Bürger vor dem Verbrechen (und dem, was sie dafür halten). In Ziele, Nutzen und Forschungsstand Viktimisierungsbefragungen in Deutschland, Bd. 1, Hrsg. N. Guzy, C. Birkel, und R. Mischkowitz, 458-487. Wiesbaden: Bundeskriminalamt.

Hirtenlehner, Helmut, Eva Groß, und Julia Meinert. 2016. Fremdenfeindlichkeit, Straflust und Furcht vor Kriminalität. Interdependenzen im Zeitalter spätmoderner Unsicherheit. Soziale Probleme 27(1):17-47.

Hooghe, Marc, und Thomas de Vroome. 2016. The relation between ethnic diversity and fear of crime: an analysis of police records and survey data in Belgian communities. International Journal of Intercultural Relations 50:66-75.

Hummelsheim, Dina, Helmut Hirtenlehner, Jonathan Jackson, und Dietrich Oberwittler. 2011. Social insecurities and fear of crime: a cross-national study on the impact of welfare state policies on crimerelated anxieties. European Sociological Review 27(3):327-345.

Hunter, Albert J. 1978. Symbols of incivility: social disorder and fear of crime in urban neighborhoods. Center for Urban Affairs, Northwestern University, Working Paper.

Jackson, Jonathan. 2004. Experience and expression. Social and cultural significance in the fear of crime. British Journal of Criminology 44(6):946-966.

Jackson, Jonathan. 2009. A psychological perspective on vulnerability in the fear of crime. Psychology, Crime \& Law 15(4):365-390.

Jackson, Jonathan. 2015. Cognitive closure and risk sensitivity in the fear of crime. Legal and Criminological Psychology 20(2):222-240.

Janssen, Heleen, Dominik Gerstner, and Dietrich Oberwittler (eingereicht). Perceptions of Disorder and the Concentration of Visible Minorities: The Moderating Role of Interethnic Contact and Xenophobic Attitudes.

Jost, John T., Jack Glaser, Arie W. Kruglanski, und Frank J. Sulloway. 2003. Political conservatism as motivated social cognition. Psychological Bulletin 129(3):339-375.

Kawakami, Kerry, David M. Amodio, und Kurt Hugenberg. 2017. Intergroup perception and cognition: an integrative framework for understanding the causes and consequences of social categorization. Advances in Experimental Social Psychology 55:1-80.

Kelling, George E., und James Q. Wilson. 1982. Broken windows: the police and neighborhood safety. Atlantic Monthly 249(3):29-38.

Kruglanski, Arie W., Antonio Pierro, Lucia Mannetti, und Eraldo De Grada. 2006. Groups as epistemic providers: need for closure and the unfolding of group-centrism. Psychological Review 113(1):84-100.

Kubrin, Charis E. 2008. Making order of disorder. A call for conceptual clarity. Criminology \& Public Policy 7(2):203-214.

LaGrange, Randy L., Kenneth F. Ferraro, und Michael Supancic. 1992. Perceived risk and fear of crime: role of social and physical incivilities. Journal of Research in Crime and Delinquency 29(3):311-334.

Lewis, Dan A., und Michael G. Maxfield. 1980. Fear in the neighborhoods: an investigation of the impact of crime. Journal of Research in Crime and Delinquency 17(2):160-189.

Link, Nathan W., M. Kelly James, Joseph R. Pitts, Kelly Waltman-Spreha, und Ralph B. Taylor. 2014. Reversing broken windows: evidence of lagged, multilevel impacts of risk perceptions on perceptions of incivility. Crime \& Delinquency 63(6):659-682.

Lüdemann, Christian. 2006. Kriminalitätsfurcht im urbanen Raum. Eine Mehrebenenanalyse zu individuellen und sozialräumlichen Determinanten von Kriminalitätsfurcht im städtischen Raum. Kölner Zeitschrift für Soziologie und Sozialpsychologie 58(2):285-306.

Markowitz, Fred E., Paul E. Bellair, Allen E. Liska, und Jianhong Liu. 2001. Extending social disorganization theory: modeling the relationships between cohesion, disorder, and fear. Criminology 39(2):293-319.

Mead, George H. 1918. The psychology of punitive justice. American Journal of Sociology 23:577-602.

van der Meer, Tom, und Jochem Tolsma. 2014. Ethnic diversity and its effects on social cohesion. Annual Review of Sociology 40:459-478.

Mellgren, Caroline, Lieven Pauwels, und M. Torstensson Levander. 2011. Neighbourhood disorder and worry about criminal victimization in the neighbourhood. International Review of Victimology 17(3):291-310.

Merton, Robert K. 1948. The self-fulfilling prophecy. The Antioch Review 8(2):193-210.

Obergfell-Fuchs, Joachim, und Helmut Kury. 1996. Sicherheitsgefühl und Persönlichkeit. Monatsschrift für Kriminologie und Strafrechtsreform 79(2):97-113. 
Oberwittler, Dietrich. 2008. Armut macht Angst. Ansätze einer sozialökologischen Interpretation der Kriminalitätsfurcht. In Soziologie sozialer Probleme und sozialer Kontrolle. Realitäten, Repräsentationen und Politik. Festschrift für Günter Albrecht, Hrsg. A. Groenemeyer, S. Wieseler, 215-230. Wiesbaden: VS.

Oberwittler, Dietrich. 2013. Wohnquartiere und Kriminalität - Überblick über die Forschung zu den sozialräumlichen Dimensionen urbaner Kriminalität. In Städtische Armutsquartiere - Kriminelle Lebenswelten? Studien zu sozialräumlichen Kontexteffekten auf Jugendkriminalität und Kriminalitätswahrnehmungen, Hrsg. D. Oberwittler, S. Rabold, und D. Baier, 45-96. Wiesbaden: Springer VS.

Oberwittler, Dietrich, und Dominik Gerstner. 2016. Kriminalitätsfurcht in großstädtischen Wohngebieten - Wie sozialräumliche Bedingungen die Unsicherheitswahrnehmungen beeinflussen. In Grenzenlose Sicherheit? Gesellschaftliche Dimensionen der Sicherheitsforschung, Hrsg. P. Zoche, S. Kaufmann, und H. Arnold, 95-116. Berlin: LIT.

Perkins, D.D., und Ralph B. Taylor. 1996. Ecological assessments of community disorder: their relationship to fear of crime and theoretical implications. American Journal of Community Psychology 24(1):63-107.

Perkins, D.D., J. Meeks, und Ralph B. Taylor. 1992. The physical environment of street blocks and resident perceptions of crime and disorder: Implications for theory and measurement. Journal of Environmental Psychology 12(1):21-34.

Pickett, Justin T., Ted Chiricos, Kristin M. Golden, und Marc Gertz. 2012. Reconsidering the relationship between perceived neighborhood racial composition and whites' perceptions of victimization risk: do racial stereotypes matter? Criminology 50(1):145-186.

Pritsch, Julian, und Dietrich Oberwittler. 2015. Kriminalitätsfurcht in Deutschland - Kontexteffekte auf ein individuelles Empfinden. In Subjektive und objektivierte Bedingungen von (Un)Sicherheit. Studien zum Barometer Sicherheit in Deutschland (BaSiD), Hrsg. R. Haverkamp, H. Arnold, 231-257. Berlin: Duncker \& Humblot.

Quillian, Lincoln, und Devah Pager. 2001. Black neighbors, higher crime? The role of racial stereotypes in evaluation of neighborhood crime. American Journal of Sociology 107(3):717-767.

Raudenbush, Stephen W., und Robert J. Sampson. 1999. Ecometrics: toward a science of assessing ecological settings, with appliance to the systematic social observation of neighborhoods. Sociological Methodology 29:1-41.

Richeson, Jennifer A., und Samuel R. Sommers. 2016. Toward a social psychology of race and race relations for the twenty-first century. Annual Review of Psychology 67:439-463.

Robinson, Jennifer B., Brian A. Lawton, Ralph B. Taylor, und Douglas D. Perkins. 2003. Multilevel longitudinal impacts of incivilities: fear of crime, expected safety, and block satisfaction. Journal of Quantitative Criminology 19(3):237-274.

Ross, Catherine, und Sun J. Jang. 1999. Neighborhood disorder, fear, and mistrust: the buffering role of social ties with neighbors. American Journal of Community Psychology 28(4):401-420.

Sampson, Robert J. 2009. Disparity and diversity in the contemporary city: social (dis)order revisited. British Journal of Sociology 60(1):1-31.

Sampson, Robert J., und Stephen Raudenbush. 2004. Seeing disorder: neighborhood stigma and the social construction of „,broken windows“. Social Psychology Quarterly 67(4):319-342.

Sampson, Robert J., und Stephen W. Raudenbush. 1999. Systematic social observation of public spaces: a new look at disorder in urban neighborhoods. American Journal of Sociology 105(3):603-651.

Semyonov, Moshe, Anastasia Gorodzeisky, und Anya Glikman. 2012. Neighborhood ethnic composition and resident perceptions of safety in European countries. Social Problems 59(1):117-135.

Sessar, Klaus. 2010. Kriminalitätseinstellungen und sozialer Wandel. Gleichzeitig eine Auseinandersetzung mit Forschungen zur Verbrechensfurcht und Punitivität. Monatsschrift für Kriminologie und Strafrechtsreform 93(5):361-391.

Sessar, Klaus, Wolfgang Stangl, und René van Swaaningen (Hrsg.). 2007. Großstadtängste. Untersuchungen zu Unsicherheitsgefühlen und Sicherheitspolitiken in europäischen Kommunen. Wien, Berlin: LIT.

Skogan, Wesley G. 1990. Disorder and decline. Crime and the spiral of decay in American neighborhoods. New York: Free Press.

Skogan, Wesley G. 1995. Crime and the racial fears of white Americans. The ANNALS of the American Academy of Political and Social Science 539(1):59-71.

Skogan, Wesley G. 2015. Disorder and decline: the state of research. Journal of Research in Crime and Delinquency 52(4):464-485.

Smith, Jason Matthew. 2010. Does crime pay? Issue ownership, political opportunity, and the populist right in Western Europe. Comparative Political Studies 43(11):1471-1498. 
Sturgis, Patrick, Ian Brunton-Smith, Jouni Kuha, und Jonathan Jackson. 2013. Ethnic diversity, segregation and the social cohesion of neighbourhoods in London. Ethnic and Racial Studies 37(8):1286-1309.

Taylor, Ralph B. 2001. Breaking away from broken windows: Baltimore neighborhoods and the nationwide fight against crime, grime, fear, and decline. Boulder: Westview Press.

Taylor, Ralph B. 2015. Community criminology. New York: New York University Press.

Taylor, Ralph B., Sally Ann Shumaker, und Stephen D. Gottfredson. 1985. Neighborhood-level links between physical features and local sentiments: deterioration, fear of crime, and confidence. Journal of Architectural and Planning Research 2(4):261-275.

Visser, Mark, Marijn Scholte, und Peer Scheepers. 2013. Fear of crime and feelings of unsafety in European countries: macro and micro explanations in cross-national perspective. Sociological Quarterly 54(2):278-301.

Wickes, Rebecca, John R. Hipp, Renee Zahnow, und Lorraine Mazerolle. 2013. „Seeing“ minorities and perceptions of disorder: explicating the mediating and moderating mechanisms of social cohesion. Criminology 51(3):519-560.

Wyant, Brian R. 2008. Multilevel impacts of perceived incivilities and perceptions of crime risk on fear of crime isolating endogenous impacts. Journal of Research in Crime and Delinquency 45(1):39-64.

Yang, Sue-Ming, und Chih-Chao Pao. 2015. Do we „see the same thing“? An experimental look into the black box of disorder perception. Journal of Research in Crime and Delinquency 52(4):534-566. 